\title{
Review
}

Kidney Dis 2015;1:138-146

Received: April 14, 2015

DOI: $10.1159 / 000431214$

\section{Macrophage Phenotype in Kidney Injury and Repair}

\author{
Xiao-Ming Meng ${ }^{a}$ Patrick Ming-Kuen Tang ${ }^{b}$ Jun Li ${ }^{a}$ Hui Yao Lan ${ }^{b, c}$

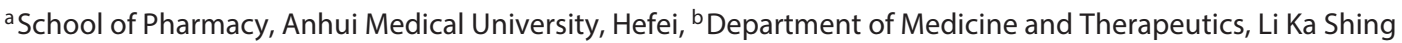 \\ Institute of Health Sciences, The Chinese University of Hong Kong, Hong Kong, SAR, and 'Shenzhen Research \\ Institute, The Chinese University of Hong Kong, Shenzhen, China
}

\section{Key Words}

Macrophage · Phenotype $\cdot$ Inflammation $\cdot$ Fibrosis

\begin{abstract}
Background: Glomerular and interstitial macrophage infiltration is a feature for both the acute and chronic kidney diseases. Macrophages have been shown to play a diverse role in kidney injury and repair. Thus, macrophages may be a key cell type in acute and chronic kidney injury and repair. Summary and Key Messages: During renal inflammation, circulating monocytes are recruited and then become activated and polarized. By adapting to the local microenvironment, macrophages can differentiate into different phenotypes and function as a double-bladed sword in different stages of kidney disease. In general, M1 macrophages play a pathogenic role in boosting inflammatory renal injury, whereas M2 macrophages exert an anti-inflammatory and wound healing (or profibrotic) role during renal repair. In this review, we highlight the phenotypic polarization of macrophages in renal diseases and dissect their distinct functions in renal injury and repair processes, respectively. Moreover, the current understanding of regulatory mechanisms on the phenotypic switch and macrophage-related therapy are also intensively discussed.

๑) 2015 S. Karger AG, Basel
\end{abstract}

\section{Introduction}

Macrophage, a critical component of the phagocytic system, was first described by Metchnikoff over 100 years ago. Studies in the early period mainly focused on their destructive properties and mechanisms by which they kill microorganisms. It is now clear that macrophages may also exert much broader effects in tissue repair and in maintaining tissue integrity [1]. Macrophages are highly heterogeneous and plastic. It is well documented that they are activated and polarized into different phenotypes in response to the local microenvironment; then, they secret different types of cytokines and growth factors accordingly after being recruited and differentiated from monocytes. The macrophages may alter the microenvironment of the diseased kidney by interacting with their neighboring cells, including immune cells, tubular epithelial cells, endothelial cells, mesangial cells, podocytes, stem cells and so on, which acts as a feedback mechanism and may determine the fate of renal diseases [2]. Until now, accumulating evidence has shown that macrophages with distinct phenotypes exert diverse effects during kidney injury and repair induced by pathogens or endogenous injury stimuli [3]. In this setting, the present review will emphasize the up-to-date information about the macro-

\section{KARGER 125}

(c) 2015 S. Karger AG, Base

2296-9381/15/0012-0138\$39.50/0

E-Mail karger@karger.com

www.karger.com/kdd
Prof. Hui Yao Lan

Li Ka Shing Institute of Health Sciences

The Chinese University of Hong Kong

Hong Kong, SAR (China)

E-Mail hylan@cuhk.edu.hk 
phage phenotypes, functions and regulatory mechanisms in different stages of kidney injury and repair. In addition, the therapeutic potential of macrophage-based and targeted therapies for renal diseases will also be evaluated.

\section{Activation and Polarization of Macrophages in Kidney Injury and Repair}

In the diseased kidney, bone marrow myeloid progenitor-derived monocytes are well accepted as the major source of infiltrated macrophages [1, 4]. Monocytes can be classified into different subsets according to the expression of lymphocyte antigen 6C (Ly6C), an antigen that represents different stages of a continuous maturation pathway, and their chemokine receptor profiles such as CCR2 and CX3CR1 [3]. For instance, CCR2 ${ }^{+} L y 6 C^{+}$ monocyte infiltrated into the sites of inflammation has been defined as a specific monocyte subset which participates in the immune response or tissue remodeling [5]. After being recruited into the injured kidney, monocytes can differentiate into macrophages with distinct activation states under the influence of the local microenvironments. To represent the Th1/Th2 paradigm, classification of M1/M2 macrophages is widely used, although it is considered to be an inadequate method for representing the diversity of the expanded phenotypes [6]. In most occasions, M1 (proinflammatory) is also called a classic activated macrophage which can be induced by interferon (IFN) $-\gamma$ and lipopolysaccharide (LPS), while alternatively activated M2 (wound healing/profibrotic) could be generated by culturing with interleukin (IL)- 4 and IL- 13 in vitro. In addition, M2 macrophages can be further subcategorized by their distinct stimuli and properties: M2a macrophages are induced by IL-4 plus IL-13; M2b macrophages are induced by immune complexes, and M2c (anti-inflammatory) subtypes are induced by IL-10 plus transforming growth factor (TGF) $\beta$ or by glucocorticoids [2] (table 1).

It is also identified that three populations of macrophages, i.e. Ly $6 C^{\text {high }}$, Ly6 $C^{\text {int }}$ and Ly6C ${ }^{\text {low }}$, are detected in the kidney, which are derived from a single population of circulating inflammatory Ly6C ${ }^{\text {high }}$ monocytes. The activated Ly6Chigh macrophages produce an abundance of chemokines (such as MIP1) and cytokines (such as IL$1 \beta$ ), mimicking the M1 phenotype; in contrast, the Ly6C low macrophages secret CCL17, platelet-derived growth factor (PDGF) and insulin-like growth factor 1 (IGF1), which are closely related to wound healing and fibrogenesis like M2 macrophages [4]. In the diseased kid-

Macrophage Phenotype in Kidney Injury and Repair ney, macrophages can be activated by many factors including cytokines produced by other immune cells (T cells, NK cells, etc.), PAMPs (pathogen-associated molecular patterns), DAMPs (damage-associated molecular patterns), and immune complexes [4, 7]. In general, kidney M1 macrophages are activated by proinflammatory cytokines such as IFN- $\gamma$, LPS and tumor necrosis factor (TNF)- $\alpha$, while M2 polarization is initiated by the Th2 cytokines. In addition, it is also believed that phagocytic uptaking of apoptotic cells may switch macrophages towards an M2 phenotype [2]. Moreover, an in vivo switch from the M1 to the M2 phenotype is also detected, which is supported by the finding that fluorescence-labeled M1 cells (IFN- $\gamma$ polarized) can gradually switch to an M2 phenotype in the repaired kidney during acute kidney injury [8].

\section{Macrophages in Kidney Injury}

Glomerular and interstitial macrophage infiltration could be detected in almost all types of acute kidney injury and progressive chronic renal diseases of both experimental models and human biopsies [1]. It was reported that a high level of inducible nitric oxide synthase (iNOS), instead of Arginase-1, can be detected in F4/80+ macrophages $24 \mathrm{~h}$ after the injury, indicating that proinflammatory M1 macrophages became predominant in the early stage of kidney diseases [8]. In addition, another study demonstrated that 2 days after rhabdomyolysis, macrophages with the $\mathrm{F} 4 / 80^{\text {low }} \mathrm{CD} 11 \mathrm{~b}^{\text {high }}$ Ly $6 \mathrm{~b}^{\text {high }}$ CD206 ${ }^{\text {low }} \mathrm{M} 1$ phenotype were mainly found in the kidney of a rhabdomyolysis-induced renal injury model [9]. It is well accepted that classic activated macrophages are capable of producing proinflammatory factors such as IL-1, TNF- $\alpha$, IL-6, IL-23, reactive oxygen species (ROS), nitric oxide, and iNOS; overproduction of these factors may induce severe kidney damage when the inflammation remains unresolved. Compelling evidence indicates that the degree of proinflammatory macrophage infiltration is strongly correlated with the severity of renal damage in both acute kidney injury and chronic kidney disease models. The pathogenic role of these proinflammatory macrophages has been evaluated by using different macrophage depletion and transfer techniques in numerous kidney disease models [10]. For instance, liposomal clodronate-induced macrophage depletion in the early stage of the kidney disease remarkably attenuates renal injury and renal fibrosis in ischemia/reperfusion injury (IRI) and rhabdomyolysis-induced kidney injury models, indicating the pathogenic role of M1 cells in the initiation of acute kidney injury $[9,11,12]$. Moreover, proinflammatory macrophages also mediate renal injury in the chron- 
Table 1. Macrophage phenotypes and functions

\begin{tabular}{|c|c|c|c|c|}
\hline & \multicolumn{4}{|l|}{ Macrophage phenotypes } \\
\hline & M1 & $\mathrm{M} 2 \mathrm{a}$ & $\mathrm{M} 2 \mathrm{~b}$ & $\mathrm{M} 2 \mathrm{c}$ \\
\hline $\begin{array}{l}\text { Surface } \\
\text { marker }\end{array}$ & $\begin{array}{l}\text { MHC II, CD16/32,CD80, } \\
\text { CD86, IL-1R, IL -12, IL-23 }\end{array}$ & $\begin{array}{l}\text { MHC II, CCR2, mannose receptor, } \\
\text { Dectin-1, DC-SIGN, MGL1/2,IL-1RII } \\
\text { YM1/2, Fizz1 }\end{array}$ & $\begin{array}{l}\text { MHC II, FcgR1, } \\
\text { CD80, CD86 }\end{array}$ & $\begin{array}{l}\text { cd150, cd163, } \\
\text { stabilin-1, IL-1RA, } \\
\text { mannose receptor }\end{array}$ \\
\hline $\begin{array}{l}\text { Secretion } \\
\text { profile }\end{array}$ & $\begin{array}{l}\text { TNF- } \alpha \text {, IL-1, IL-6, IL-12, } \\
\text { IL-23, CCL3, CCL5, } \\
\text { CXCL1, CXCL2, CXCL10, } \\
\text { ROS, iNOS, NO }\end{array}$ & $\begin{array}{l}\text { CCL13, CCL14, CCL17, CCL18, CCL22, } \\
\text { CCL23, CCL24, CCL26, TGF- } \beta \text {, PDGF, } \\
\text { fibronectin, bIG-H3, IGF-1, MMP-9, } \\
\text { MMP-12, Arginase-1, }\end{array}$ & $\begin{array}{l}\text { IL-1, IL-6, TNF- } \alpha \text {, } \\
\text { IL-10, IL-12 }\end{array}$ & $\begin{array}{l}\text { IL-10, TGF- } \beta \text {, } \\
\text { mannose receptor, } \\
\text { Arginase- } 1\end{array}$ \\
\hline $\begin{array}{l}\text { Phenotypic } \\
\text { function }\end{array}$ & $\begin{array}{l}\text { Host defense, } \\
\text { proinflammatory Th1 } \\
\text { immune response }\end{array}$ & $\begin{array}{l}\text { Anti-inflammatory Th2 immune } \\
\text { response, wound healing, profibrotic }\end{array}$ & $\begin{array}{l}\text { Immunoregulation, } \\
\text { Th2 activation }\end{array}$ & $\begin{array}{l}\text { Immunosuppression, } \\
\text { matrix remodeling, } \\
\text { tissue repair }\end{array}$ \\
\hline
\end{tabular}

ic kidney disease model, as supported by the finding that depletion/deactivation of macrophages by the c-fms inhibitor or JNK inhibitor halts the development of crescentic anti-GBM glomerulonephritis [13]. By contrast, adoptive transfer of bone marrow-derived or NR8383 macrophages at an early stage aggravates renal injury in the same disease model [10]. Collectively, proinflammatory macrophages (M1) can accelerate renal injury possibly through the following mechanisms: (1) boosting the inflammatory response by releasing an abundance of chemokines and proinflammatory cytokines [10]; (2) macrophage-derived ROS and TNF- $\alpha$ can trigger apoptosis of renal cells, therefore accelerating the renal injury [14], and (3) overproduction of profibrotic cytokines and growth factors by macrophages may induce abnormal wound healing and may finally lead to renal fibrosis [2] (fig. 1).

\section{Macrophages in Kidney Repair}

The anti-inflammatory and reparative roles of macrophages have recently attracted attention $[8,11,15]$. It has been identified that macrophages with the M2 phenotype are predominant in the repair process of kidney disease models including IRI and unilateral ureteral obstruction (UUO) nephropathy, as a key regulator for anti-inflammation and wound healing [8]. By using a fluorescencelabeled cell-tracing technique, the results from a recent study indicated that the majority of F4/80+ cells began to lose the iNOs markers and express a high level of Arginase- 1 on the 6 th day after IRI, indicating the polarization of macrophages towards $\mathrm{M} 2$ in the repair phase of acute kidney injury [8]. The functional role of these M2 macrophages in the renal repair process has also been explored. Depletion of macrophages in the late stage of the IRI model slows down the proliferation of tubular epithelial cells and delays the renal repair process, while transfusion of IL-4-polarized M2 macrophages accelerates the repair [16]. Mechanically, M2 cells exert an anti-inflammatory effect mainly through their high endocytic capacities and production of anti-inflammatory factors [3]; when the unwanted cells and debris are engulfed, macrophages will release an abundance of IL-10 together with other M2secreted anti-inflammatory cytokines and trophic factors, such as TGF- $\beta$, IGF and hepatocyte growth factor. M2 macrophages are also able to block the activation of $\mathrm{T}$ cells and macrophages, therefore suppressing renal inflammation. Additionally, M2c macrophages, rather than other subsets, can induce the production of regulatory $\mathrm{T}$ cells to exert their immunosuppressive effects [17]. M2 macrophages are capable of inducing angiogenesis and accelerate the repair of the endothelial injury. Moreover, recent studies demonstrated that macrophage-derived Wnt7b signaling can directly stimulate epithelial responses or accelerate the renewal of stem or progenitor cells, thereby promoting kidney repair following IRI [18].

It is identified that anti-inflammatory macrophages can enhance tubular reepithelialization through producing trophic factors; however, interstitial fibrosis will be initiated when the inflammation is persistent or too severe [2]. Results from a recent study also suggested that 


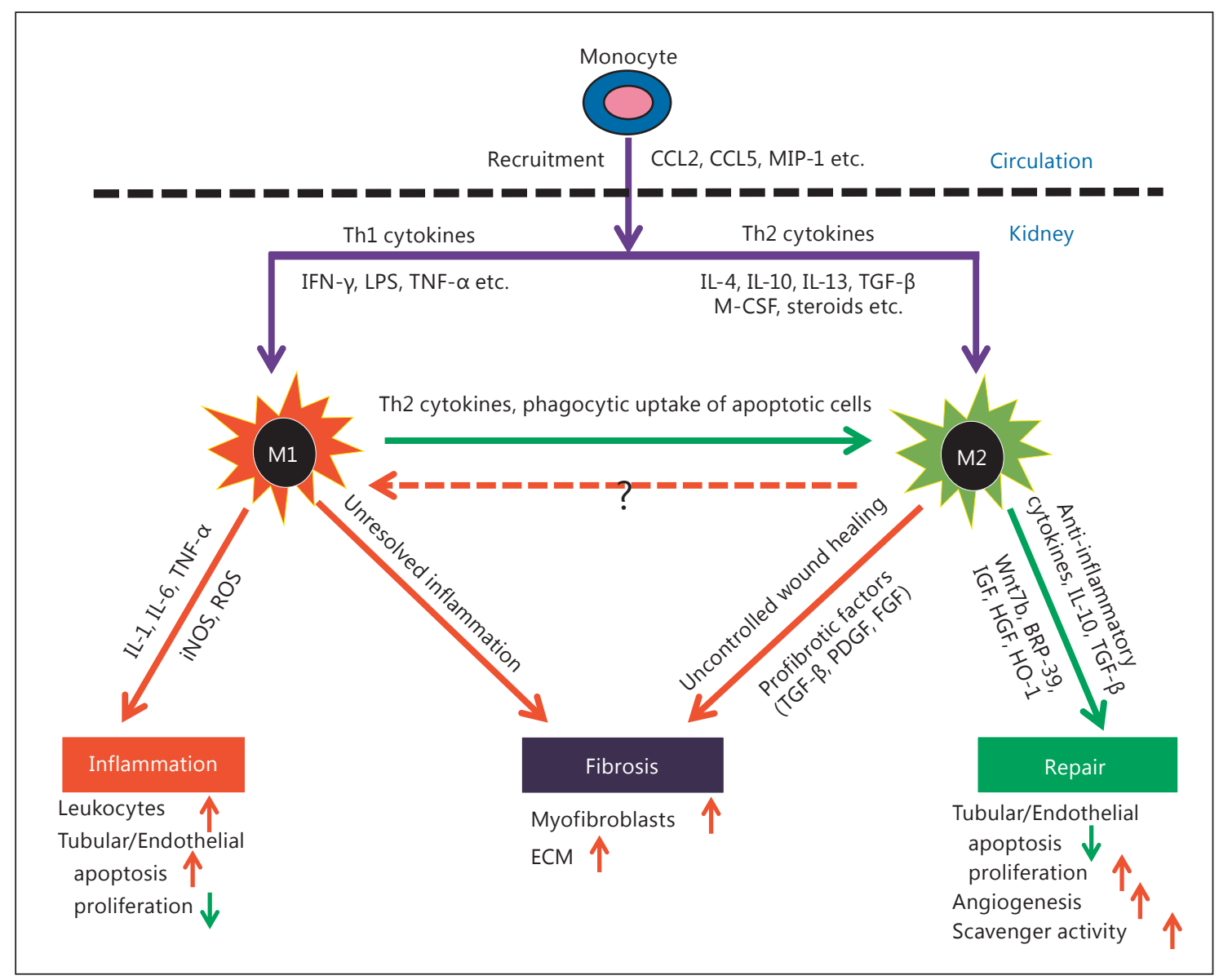

Fig. 1. Macrophage phenotypes in renal injury and repair. During renal inflammation, circulating monocytes are recruited into the injured kidney in response to chemokines released by immune cells and intrinsic kidney cells. Then, monocytes differentiate and polarize into macrophages with distinct phenotypes under the influence of local microenvironments. Th1 cytokines (such as IFN- $\gamma$ and TNF- $\alpha$ ) induce M1 macrophages, while Th2 cytokines (such as IL-4, IL-13, and IL-10) promote M2 polarization. Additionally, M1 can switch to M2 macrophages in the presence of Th2 cytokines or after engulfing apoptotic cells. M1 macrophages produce

the failure of macrophages to switch from a proinflammatory M1 to a reparative M2 phenotype may lead to progressive renal inflammation and fibrosis after IRI [19] (fig. 1).

\section{Macrophages in Kidney Fibrosis and Fibrolysis}

As shown in figure 1, uncontrolled wound healing and unresolved renal injury can lead to renal fibrosis. Accumulating evidence shows that deletion of macrophage an abundance of proinflammatory cytokines (IL-1, IL-6, and TNF- $\alpha$ ), iNOS, and ROS, therefore promoting renal injury. M2 macrophages can synthesize anti-inflammatory cytokines (such as IL-10 and TGF- $\beta$ ) and trophic factors (such as hepatocyte growth factor and IGF) to accelerate the resolution of inflammation and repair process. However, unresolved inflammation and uncontrolled wound healing processes can trigger the process of renal fibrosis by enhancing activation and differentiation of macrophages. can reduce renal fibrosis in most occasions, which implies the profibrotic property of macrophages in various renal diseases [20]. For instance, depletion of macrophages with liposome clodronate attenuates renal fibrosis following ischemia/reperfusion-induced acute kidney injury [12], which is further evidenced by the findings that inhibition of MCP-1 by delivery of a human MCP-1 mutant with $\mathrm{N}$-terminal deletion could largely attenuate renal fibrosis. In addition, large numbers of M2 macrophages can be observed in the active fibrotic area in the renal biopsy of patients with IgA nephropathy and are 
strongly correlated with the severity of glomerulosclerosis and interstitial fibrosis [20]. Consistently, administration of glucocorticoid promotes global glomerulosclerosis in rat Thy-1 mesangial proliferative glomerulonephritis, which is associated with numbers of macrophages with the M2 phenotype [21]. Moreover, a recent study indicated that macrophages can be switched from the M1 to the M2 phenotype during the progression of chronic fibrosis, whereas elimination of macrophages in the fibrotic phase inhibits renal fibrosis by blocking the TGF- $\beta_{1}$ expression and capillary rarefaction [22]. Taken together, macrophages can promote renal fibrosis through several mechanisms as follows: (1) M2 macrophages can secret numbers of profibrotic factors, including TGF- $\beta_{1}$, fibroblast growth factor (FGF)-2, PDGF, and galectin-3 (a $\beta$-galactosidase-binding lectin), which promotes myofibroblast proliferation, survival and activation, resulting in the overproduction of extracellular matrix (ECM), although a recent study indicated that macrophage-derived TGF- $\beta_{1}$ may not be indispensable in UUO-induced renal interstitial fibrosis [23]. (2) Macrophages can produce a high amount of cytokines and factors, such as IL-1, matrix metalloproteinase (MMP)9, TGF- $\beta_{1}$, angiotensin II, PDGF, IGF-1, and FGF-2, thereby enhancing the transition process of kidney resident cells to collagen-producing myofibroblasts, such as epithelial-mesenchymal transition, endothelial-mesenchymal transition, and local activation of pericytes, fibroblasts, and mesangial cells [24]. In addition to the fact that macrophages can synthesize fibronectin and collagen in response to a profibrotic microenvironment, evidence also indicated that macrophages could transdifferentiate into collagen-producing fibrocytes [4]. (3) The activated macrophages are capable of destroying glomerular and peritubular capillaries, and therefore promoting hypoxia-driven fibrosis [22].

Although the origin of myofibroblasts in renal fibrosis is still under debate, there is evidence that considerable myofibroblasts may be transdifferentiated by bone marrow-derived macrophages besides other sources, such as resident fibroblasts, pericytes, endothelial cells, epithelial cells, and fibrocytes [25]. It has been clearly demonstrated that cultured macrophages can be induced into a-SMA+ cells, which may produce collagens [26]. Moreover, our recent study identified numerous CD68+a-SMA+ cells in human biopsies with progressive renal fibrosis but not in nonfibrotic kidneys such as in patients with minimalchange disease [25], suggesting macrophage-myofibroblast transition during the development of renal fibrosis. The phenomenon of macrophage-myofibroblast transi- tion was further confirmed in UUO nephropathy, where substantial F4/80+ $\alpha$-SMA+ cells can be detected in the fibrotic lesions of the obstructive kidneys. Taken together, the transition of macrophages into myofibroblasts may be one of the mechanisms by which inflammatory macrophages promote renal fibrosis [20], although the detailed mechanisms controlling this process remain to be determined.

However, emerging evidence illustrates that the M2 macrophages may not necessarily cause renal fibrosis directly [2]. When wound healing is initiated, inflammation and epithelial healing will occur as the first-line response to the injury; then, the major event of second-line response fibrogenesis may be followed only if the epithelial healing is insufficient and incomplete due to ongoing injury and unresolved renal inflammation [20], indicating that the balance between the efficiency of tubular regeneration and the severity and persistence of tubular damage may be a key factor leading to renal fibrosis.

In addition, macrophages are also identified as a negative regulator for renal fibrosis in the fibrolysis stage [2]. Although the exact phenotype of fibrolytic macrophages is still not fully characterized, emerging evidence shows that these cells may participate in the resolution of renal fibrosis through secreting MMPs which can degrade the ECM in the fibrotic kidney $[2,27]$. The regression of established organ fibrosis is well explored in the liver, as conditional knockout of macrophages in the late stage of CCL4-induced liver fibrosis delayed the resolution of liver scars, which may be due to the absence of macrophageinduced hepatic stellate cell apoptosis [28]. Evidence for the negative regulatory role of macrophages in renal fibrosis has been collected in a reversible obstructive kidney disease model [27]. It is also identified that deficiency of urokinase-type plasminogen activator receptor inhibits macrophage infiltration and reduces the scavenger receptor function, thereby impairing their clearance effects and promoting renal fibrosis [29]. Moreover, it is demonstrated that an adoptive transfer of macrophages in the fibrotic kidney at a late stage of UUO nephropathy (day 14 after surgery), instead of at an early stage (day 5 after surgery), leads to reduced renal fibrosis and enhances renal repair possibly by degrading ECM components in an MMP-2-dependent manner [30]. Of note, the functions of MMPs are distinct and complex in different stages of fibrosis. For example, MMP-2 and MMP-9 are proven to be pathogenic in the early stage of renal disease due to their matrix lysis activities to damage the integrity of glomerular and tubular basement membranes and induce epithelial-mesenchymal transition [31]. 
Regulatory Mechanisms of Macrophage Polarization in Kidney Diseases

Further investigation on the mechanisms of activation and polarization of macrophages at the molecular level may lead to the discovery of novel therapeutic targets for renal diseases. In addition to the classic regulatory pathways and factors such as NF- $\kappa B$, JNK, JAK/STAT, PI3K/ AKT and Notch signaling pathways, a number of novel molecules and signaling pathways have recently been identified to regulate macrophage polarization in kidney diseases, which is discussed below.

For instance, a recent study identified that the release of high-mobility group box 1 protein from tubular epithelial cells and infiltrated macrophages can contribute to the M1 macrophage transition, as it largely induces iNOS while suppressing the production of IL-10 in macrophages. Blockade of the release of high-mobility group box 1 with a glycyrrhizic acid derivative attenuates UUOmediated kidney injury and fibrosis [32]. Suppressor of cytokine signaling-3 (SOCS-3), an important intracellular negative regulator of several signaling pathways, is found to be highly expressed in renal proximal tubules in response to acute kidney injury. Conditional knockout of SOCS-3 from proximal tubular cells significantly enhances their proliferation during the repair process due to the loss of an antimitotic effect of SOCS-3 via a JAK/STATdependent mechanism. Moreover, it is also identified that the deficiency of SOCS-3 results in an increase in F4/80+ cells with a predominant M2 phenotype. In contrast, overexpression of SOCS-3 in epithelial cells largely induces classical activation of cocultured macrophages, indicating its role in mediating the switch from M1 to M2 [33].

The factors mediating M2 polarization are suggested to be potential therapeutic targets for renal injury. Colony-stimulating factor-1 (CSF-1) has been regarded as an important inducer of macrophage polarization. It is reported that deficiency of CSF-1 suppresses the proliferation of tubular epithelial cells and tubular repair through reducing the number of M2 macrophages [34]. Evidence indicates that CSF-1 remarkably increases the number of $\mathrm{CD} 11 \mathrm{~b}+\mathrm{F} 4 / 80+\mathrm{CD} 163+\mathrm{CD} 206^{\text {high }} \mathrm{M} 2$ cells, thereby promoting the elimination of renal crystals in hyperoxaluric mice [35]. In addition, CSF-1 also directly targets tubular epithelial cells in a macrophage-independent manner [36]. It has been well demonstrated that peripheral Ly6C ${ }^{\text {high }}$ monocytes tend to differentiate into proinflammatory M1 macrophages under granulocyte macrophage-CSF conditions. However, a very recent study identified that granulocyte macrophage-CSF can induce macrophages with a unique alternative activation profile in vivo, which differs from the canonical alternative activation induced by IL-4-triggered STAT6 signaling. This subpopulation can promote tubular proliferation and kidney tissue repair, as STAT6 is regarded as a critical transcription factor by regulating the M2 transcription effectors such as arginase- 1 and Ym1 [37]. In addition, this phenotype is detected in macrophages isolated from the repair phase of injured kidneys in a murine ischemic/reperfusion model, and is reproduced in vitro by treatment of the bone marrow-derived macrophages with conditioned media from the serum-starved tubular epithelial cells [37]. A recent work identified IL-25 as a new cytokine for promoting M2 polarization both in vivo and in vitro since the administration of IL-25 protects against renal injury in Adriamycin nephropathy at least partly dependent on IL-4/IL-13 [38]. Netrin-1, an anti-inflammatory molecule produced in tubular epithelial cells of ischemia/reperfusion kidney, is capable of suppressing monocyte migration and function in acute and chronic inflammatory diseases by targeting chemokines and NF- $\kappa B$ signaling. Netrin-1 might induce the polarization of macrophages into M2 phenotypes. This is supported by the observation that netrin- 1 transgenic mice show an increase in the number of M2 macrophages associated with enhanced expression of IL-4, IL-13, and arginase- 1 in the spleen. Furthermore, netrin- 1 can also promote M2 polarization by targeting the peroxisome proliferator-activated receptor pathway, a critical regulator in macrophage polarization [39]. Furthermore, p53 is a newly identified potential regulator of macrophage polarization, as global knockout or deletion of p53 from bone marrow enhances renal injury and impairs the renal repair process associated with the failure to induce KLF4 expression and M2 polarization [40]. Moreover, it is well accepted that paracrines from mesenchymal stem cells (MSCs) can partly contribute to their protective role in renal acute injury as evidenced by the finding that the administration of MSCs attenuates rhabdomyolysis-induced tubular injury and deterioration of renal function by increasing M2 macrophages. This is also supported by the in vitro finding that macrophages stimulated with MSCs show an increase in the M2 phenotype, and the transfer of MSC-cocultured macrophages into the macrophage depletion mice inhibits renal injury [41]. Consistently, the role of MSCs in M2 polarization was also confirmed in IRI [42]. 


\section{Macrophage-Based or Targeted Therapy for Renal Diseases}

The potential of macrophage-based therapies in renal diseases has been extensively investigated. The current studies mainly focus on the following scientific issues: (1) can macrophages be utilized as a therapeutic target in renal diseases? and (2) can macrophages serve as a therapeutic agent for treating renal diseases?

A number of studies have demonstrated that inhibition or deletion of macrophage infiltration or genetically altering the activation or phenotype of macrophages is capable of attenuating renal diseases. It has been reported that DNA vaccination or neutralizing antibody-based inhibition of chemokines, such as CCL2 and CCL5, can significantly reduce macrophage recruitment and kidney damage in Adriamycin nephropathy, nephrotoxic serum nephritis and anti-Thy-1.1 nephritis [43]. In addition, blocking CX3CR1 or intercellular adhesion molecule-1 may protect against crescentic glomerulonephritis or nephrotoxic nephritis. Moreover, anti-macrophage serum-induced deletion of macrophages has been shown to alleviate kidney injury in experimental glomerulonephritis [43]. Blockade of c-fms, a receptor of CSF, significantly reduces the proliferation and numbers of infiltrated macrophages, thereby suppressing renal inflammation and injury [44]. In addition, although deletion of macrophages using liposomal clodronate is classic and convenient and is capable of removing macrophages and reducing renal fibrosis, it is less specific [45]. Furthermore, conditional knockout CD11b-positive cells with the gene modification technique may block the deterioration of crescentic glomerulonephritis [46], although CD11b is also expressed by other phagocytic leukocytes [43]. It has been reported that inhibition of NF- $\mathrm{kB}$ by transferring antisense oligonucleotides or its natural inhibitor I $\mathrm{B}$ attenuates kidney injury, which is associated with the inhibition of the classic activation of macrophages with an increase in the number of anti-inflammatory macrophages [47]. Emerging evidence also indicates that IL-25 is likely to induce IL-4/IL-13-dependent M2 polarization in the diseased kidney to eliminate renal injury in Adriamycin nephropathy [38].

Modification of the macrophage phenotype has also been shown to be able to inhibit renal diseases in a series of studies. For instance, adoptive transfer with the IL-4and IL-13-polarized splenic macrophages to SCID mice has been shown to inhibit Adriamycin nephropathy [48]. The renoprotective effect of the ex vivo polarized macrophages was further confirmed in diabetic models [49]. It should be pointed out that the therapeutic efficiency in different M2 macrophage subsets varies. IL-10- and TGF$\beta$-induced M2c macrophages have greater potency in decreasing renal damage and proteinuria, since $\mathrm{M} 2 \mathrm{c}$ can induce the differentiation of immune-suppressing $\mathrm{T}$ regulatory cells through a B7-H4-dependent mechanism [17]. It is interesting that IL-10/TGF- $\beta$ - or IL-4/IL-13-modified bone marrow-derived macrophages fail to protect against renal injury, because the anti-inflammatory phenotype of bone marrow-differentiated M2 cells is easily lost due to the capacity of continuous proliferation [50], which minimizes the clinical application of autologous macrophage-based therapy by modifying the bone marrow cells of patients.

\section{Conclusion and Perspective}

Collectively, macrophages are polarized into distinct phenotypes in response to the local microenvironments after being recruited into the injured kidney. Macrophages with different activation stages exert diverse or even opposite effects in the development of renal disease. M1 macrophages trigger kidney injury at an early stage by releasing proinflammatory cytokines, while M2 macrophages promote resolution of renal inflammation and accelerate renal repair during the late stage of renal injury. However, M2 macrophages may also drive renal fibrosis when inflammation and wound healing are unsolved. In this setting, the therapeutic potential for kidney diseases by regulating the macrophage recruitment, activation, polarization and phenotype switch need to be extensively explored.

\section{Acknowledgements}

This study was supported by the Major State Basic Research Development Program of China (973 program, 2012CB517705), the Research Grant Council of Hong Kong (CUHK3/CRF/12R, T12-402/13-N), the Focused Investment Scheme A from The Chinese University of Hong Kong (H.Y.L.), and the National Natural Science Foundation of China (No. 81300580).

\section{Disclosure Statement}

All the authors declare that they have no competing interests. 


\section{References}

1 Wilson HM, Walbaum D, Rees AJ: Macro- 15 Huen SC, Cantley LG: Macrophage-mediated phages and the kidney. Curr Opin Nephrol Hypertens 2004;13:285-290.

-2 Anders HJ, Ryu M: Renal microenvironments and macrophage phenotypes determine progression or resolution of renal inflammation and fibrosis. Kidney Int 2011;80:915-925.

-3 Ricardo SD, van Goor H, Eddy AA: Macrophage diversity in renal injury and repair. J Clin Invest 2008;118:3522-3530.

4 Duffield JS: Macrophages and immunologic inflammation of the kidney. Semin Nephrol 2010;30:234-254.

5 Geissmann F, Jung S, Littman DR: Blood monocytes consist of two principal subsets with distinct migratory properties. Immunity 2003;19:71-82.

-6 Murray PJ, Allen JE, Biswas SK, Fisher EA, Gilroy DW, Goerdt S, Gordon S, Hamilton JA, Ivashkiv LB, Lawrence T, Locati M, Mantovani A, Martinez FO, Mege JL, Mosser DM, Natoli G, Saeij JP, Schultze JL, Shirey KA, Sica A, Suttles J, Udalova I, van Ginderachter JA, Vogel SN, Wynn TA: Macrophage activation and polarization: nomenclature and experimental guidelines. Immunity 2014;41:14-20.

7 Anders HJ: Toll-like receptors and danger signaling in kidney injury. J Am Soc Nephrol 2010;21:1270-1274.

8 Lee S, Huen S, Nishio H, Nishio S, Lee HK, Choi BS, Ruhrberg C, Cantley LG: Distinct macrophage phenotypes contribute to kidney injury and repair. J Am Soc Nephrol 2011;22: 317-326.

-9 Belliere J, Casemayou A, Ducasse L, ZakaroffGirard A, Martins F, Iacovoni JS, GuilbeauFrugier C, Buffin-Meyer B, Pipy B, Chauveau D, Schanstra JP, Bascands JL: Specific macrophage subtypes influence the progression of rhabdomyolysis-induced kidney injury. J Am Soc Nephrol 2015;26:1363-1377.

10 Cao Q, Wang Y, Harris DC: Pathogenic and protective role of macrophages in kidney disease. Am J Physiol Renal Physiol 2013; 305:F3-F11.

11 Day YJ, Huang L, Ye H, Linden J, Okusa MD: Renal ischemia-reperfusion injury and adenosine $2 \mathrm{a}$ receptor-mediated tissue protection: role of macrophages. Am J Physiol Renal Physiol 2005;288:F722-F731.

-12 Ko GJ, Boo CS, Jo SK, Cho WY, Kim HK: Macrophages contribute to the development of renal fibrosis following ischaemia/reperfusion-induced acute kidney injury. Nephrol Dial Transplant 2008;23:842-852.

13 Han Y, Ma FY, Tesch GH, Manthey CL, Nikolic-Paterson DJ: c-fms blockade reverses glomerular macrophage infiltration and halts development of crescentic anti-GBM glomerulonephritis in the rat. Lab Invest 2011;91: 978-991.

14 Kluth DC, Erwig LP, Rees AJ: Multiple facets of macrophages in renal injury. Kidney Int 2004;66:542-557. injury and repair after ischemic kidney injury. Pediatr Nephrol 2015;30:199-209.

16 Vinuesa E, Hotter G, Jung M, HerreroFresneda I, Torras J, Sola A: Macrophage involvement in the kidney repair phase after ischaemia/reperfusion injury. J Pathol 2008; 214:104-113.

17 Lu J, Cao Q, Zheng D, Sun Y, Wang C, Yu X, Wang Y, Lee VW, Zheng G, Tan TK, Wang X, Alexander SI, Harris DC: Discrete functions of M2a and M2c macrophage subsets determine their relative efficacy in treating chronic kidney disease. Kidney Int 2013;84:745-755.

18 Lin SL, Li B, Rao S, Yeo EJ, Hudson TE, Nowlin BT, Pei H, Chen L, Zheng JJ, Carroll TJ, Pollard JW, McMahon AP, Lang RA, Duffield JS: Macrophage Wnt7b is critical for kidney repair and regeneration. Proc Natl Acad Sci USA 2010;107:4194-4199.

19 Lech M, Grobmayr R, Ryu M, Lorenz G, Hartter I, Mulay SR, Susanti HE, Kobayashi KS, Flavell RA, Anders HJ: Macrophage phenotype controls long-term AKI outcomes - kidney regeneration versus atrophy. J Am Soc Nephrol 2014;25:292-304.

20 Meng XM, Nikolic-Paterson DJ, Lan HY: Inflammatory processes in renal fibrosis. Nat Rev Nephrol 2014;10:493-503.

21 Ikezumi Y, Suzuki T, Karasawa T, Hasegawa H, Kawachi H, Nikolic-Paterson DJ, Uchiyama M: Contrasting effects of steroids and mizoribine on macrophage activation and glomerular lesions in rat thy-1 mesangial proliferative glomerulonephritis. Am J Nephrol 2010;31:273-282.

22 Han Y, Ma FY, Tesch GH, Manthey CL, Nikolic-Paterson DJ: Role of macrophages in the fibrotic phase of rat crescentic glomerulonephritis. Am J Physiol Renal Physiol 2013; 304:F1043-F1053.

23 Huen SC, Moeckel GW, Cantley LG: Macrophage-specific deletion of transforming growth factor-betal does not prevent renal fibrosis after severe ischemia-reperfusion or obstructive injury. Am J Physiol Renal Physiol 2013;305:F477-F484.

24 LeBleu VS, Taduri G, O’Connell J, Teng Y, Cooke VG, Woda C, Sugimoto H, Kalluri R: Origin and function of myofibroblasts in kidney fibrosis. Nat Med 2013;19:1047-1053.

25 Nikolic-Paterson D, Wang S, Lan HY: Macrophages promote renal fibrosis through direct and indirect mechanisms. Kidney Int Suppl 2014;4:34-38.

26 Pilling D, Gomer RH: Differentiation of circulating monocytes into fibroblast-like cells. Methods Mol Biol 2012;904:191-206.

27 Ronco P, Chatziantoniou C: Matrix metalloproteinases and matrix receptors in progression and reversal of kidney disease: therapeutic perspectives. Kidney Int 2008;74:873-878.
28 Duffield JS, Forbes SJ, Constandinou CM, Clay S, Partolina M, Vuthoori S, Wu S, Lang $\mathrm{R}$, Iredale JP: Selective depletion of macrophages reveals distinct, opposing roles during liver injury and repair. J Clin Invest 2005;115: 56-65.

29 Zhang G, Kim H, Cai X, Lopez-Guisa JM, Carmeliet P, Eddy AA: Urokinase receptor modulates cellular and angiogenic responses in obstructive nephropathy. J Am Soc Nephrol 2003;14:1234-1253.

30 Nishida M, Okumura Y, Ozawa S, Shiraishi I, Itoi T, Hamaoka K: MMP-2 inhibition reduces renal macrophage infiltration with increased fibrosis in UUO. Biochem Biophys Res Commun 2007;354:133-139.

-31 Ronco P, Lelongt B, Piedagnel R, Chatziantoniou C: Matrix metalloproteinases in kidney disease progression and repair: a case of flipping the coin. Semin Nephrol 2007;27:352362.

32 Tian S, Zhang L, Tang J, Guo X, Dong K, Chen SY: HMGB1 exacerbates renal tubulointerstitial fibrosis through facilitating M1 macrophage phenotype at the early stage of obstructive injury. Am J Physiol Renal Physiol 2015; 308:F69-F75

33 Susnik N, Sorensen-Zender I, Rong S, von Vietinghoff S, Lu X, Rubera I, Tauc M, Falk CS, Alexander WS, Melk A, Haller H, Schmitt $\mathrm{R}$ : Ablation of proximal tubular suppressor of cytokine signaling 3 enhances tubular cell cycling and modifies macrophage phenotype during acute kidney injury. Kidney Int 2014; 85:1357-1368.

-34 Zhang MZ, Yao B, Yang S, Jiang L, Wang S, Fan X, Yin H, Wong K, Miyazawa T, Chen J, Chang I, Singh A, Harris RC: CSF-1 signaling mediates recovery from acute kidney injury. J Clin Invest 2012;122:4519-4532.

- 35 Taguchi K, Okada A, Kitamura H, Yasui T, Naiki T, Hamamoto S, Ando R, Mizuno K, Kawai N, Tozawa K, Asano K, Tanaka M, Miyoshi I, Kohri K: Colony-stimulating factor-1 signaling suppresses renal crystal formation. J Am Soc Nephrol 2014;25:1680-1697.

36 Menke J, Iwata Y, Rabacal WA, Basu R, Yeung YG, Humphreys BD, Wada T, Schwarting A, Stanley ER, Kelley VR: CSF-1 signals directly to renal tubular epithelial cells to mediate repair in mice. J Clin Invest 2009;119:23302342.

37 Huen SC, Huynh L, Marlier A, Lee Y, Moeckel GW, Cantley LG: GM-CSF promotes macrophage alternative activation after renal ischemia/reperfusion injury. J Am Soc Nephrol 2015;26:1334-1345.

38 Cao Q, Wang C, Zheng D, Wang Y, Lee VW Wang YM, Zheng G, Tan TK, Yu D, Alexander SI, Harris DC: IL-25 induces M2 macrophages and reduces renal injury in proteinuric kidney disease. J Am Soc Nephrol 2011; 22:1229-1239. 
39 Ranganathan PV, Jayakumar C, Ramesh G: Netrin-1-treated macrophages protect the kidney against ischemia-reperfusion injury and suppress inflammation by inducing M2 polarization. Am J Physiol Renal Physiol 2013;304:F948-F957.

40 Sutton TA, Hato T, Mai E, Yoshimoto M, Kuehl S, Anderson M, Mang H, Plotkin Z, Chan RJ, Dagher PC: p53 is renoprotective after ischemic kidney injury by reducing inflammation. J Am Soc Nephrol 2013;24:113124.

-41 Geng Y, Zhang L, Fu B, Zhang J, Hong Q, Hu J, Li D, Luo C, Cui S, Zhu F, Chen X: Mesenchymal stem cells ameliorate rhabdomyolysis-induced acute kidney injury via the activation of M2 macrophages. Stem Cell Res Ther 2014;5:80.

42 Wise AF, Williams TM, Kiewiet MB, Payne NL, Siatskas C, Samuel CS, Ricardo SD: Human mesenchymal stem cells alter macrophage phenotype and promote regeneration via homing to the kidney following ischemiareperfusion injury. Am J Physiol Renal Physiol 2014;306:F1222-F1235.
43 Wang Y, Harris DC: Macrophages in renal disease. J Am Soc Nephrol 2011;22:21-27.

44 Lim AK, Ma FY, Nikolic-Paterson DJ, Thomas MC, Hurst LA, Tesch GH: Antibody blockade of $\mathrm{c}$-fms suppresses the progression of inflammation and injury in early diabetic nephropathy in obese $\mathrm{db} / \mathrm{db}$ mice. Diabetologia 2009;52:1669-1679.

45 Kitamoto K, Machida Y, Uchida J, Izumi Y, Shiota M, Nakao T, Iwao H, Yukimura T, Nakatani T, Miura K: Effects of liposome clodronate on renal leukocyte populations and renal fibrosis in murine obstructive nephropathy. J Pharmacol Sci 2009;111:285-292.

46 Duffield JS, Tipping PG, Kipari T, Cailhier JF, Clay S, Lang R, Bonventre JV, Hughes J: Conditional ablation of macrophages halts progression of crescentic glomerulonephritis. Am J Pathol 2005;167:1207-1219.
-47 Wilson HM, Chettibi S, Jobin C, Walbaum D, Rees AJ, Kluth DC: Inhibition of macrophage nuclear factor-kappaB leads to a dominant anti-inflammatory phenotype that attenuates glomerular inflammation in vivo. Am J Pathol 2005; 167:27-37.

48 Wang Y, Wang YP, Zheng G, Lee VW, Ouyang L, Chang DH, Mahajan D, Coombs J, Wang YM, Alexander SI, Harris DC: Ex vivo programmed macrophages ameliorate experimental chronic inflammatory renal disease. Kidney Int 2007;72:290-299.

49 Parsa R, Andresen P, Gillett A, Mia S, Zhang XM, Mayans S, Holmberg D, Harris RA: Adoptive transfer of immunomodulatory M2 macrophages prevents type 1 diabetes in nod mice. Diabetes 2012;61:2881-2892.

50 Cao Q, Wang Y, Zheng D, Sun Y, Wang C, Wang XM, Lee VW, Zheng G, Tan TK, Wang YM, Alexander SI, Harris DC: Failed renoprotection by alternatively activated bone marrow macrophages is due to a proliferation-dependent phenotype switch in vivo. Kidney Int 2014;85:794-806. 